\title{
Sentimiento de autoeficacia en una muestra de profesores chilenos desde las perspectivas de género y experiencia*
}

\author{
Analysis of the self-efficacy sense in Chilean teachers from gender \\ and experience perspectives
}

\begin{abstract}
Sentimento de autoeficácia desde as perspectivas de gênero e experiência em uma amostra de professores chilenos
\end{abstract}

Carmen Gloria Covarrubias, ${ }^{a}$ Michelle Mendoza Lira. ${ }^{b}$

a Universidad de Talca. Fono: 71-2201722. Correo electrónico: cacovarrubias@utalca.cl

b Universidad Autónoma de Chile. Fono: 2-23036500. Correo electrónico: mmendozal@uautonoma.cl

\begin{abstract}
RESUMEN
En atención a las complejas demandas emanadas de la escuela, la política y la sociedad, el artículo que a continuación se presenta tiene el propósito de describir los sentimientos de autoeficacia, en los desempeños profesionales, de una muestra de 544 profesores chilenos. Los estudios del constructo, realizados en casi tres décadas, no sólo han intentado explicar la realidad educativa, sino que también han valorado el importante papel que tiene en las prácticas pedagógicas. Conforme a ello, se ha realizado la traducción y la adaptación del cuestionario "Teacher Sense of Efficacy Scale" (TSES) de Tschannen-Moran \& Woolfolk (2001). Cabe señalar que el proceso de validación ha revelado índices de interés tanto para su estructura factorial como para su fiabilidad. Los resultados, por su parte, han demostrado que el género no es una variable que altere los sentimientos de autoeficacia docente, a diferencia de la experiencia docente que sí ha reportado diferencias significativas.
\end{abstract}

Palabras clave: educación, autoeficacia, profesores, género, experiencia.

\begin{abstract}
During the past three decades, research studies regarding the theory of teachers' self-efficacy not only have tried to explain the teaching context and meet the demands set by the schools, politics and society, but they have also positioned the important value that this concept has in the teaching practices. The objective of the current study is to describe the feelings of self-efficacy in the teaching practices of an overall sample consisting of 544 Chilean teachers. So as to achieve such purpose, we translated and adapted the questionnaire entitled "Teacher Sense of Efficacy Scale" (TSES) created by Tschannen-Moran \& Woolfolk (2001). We should also emphasize the fact that the validation process revealed high interest indexes for both its factorial experience and its reliability. On the other hand, the findings suggest that gender is not a variable affecting these results, unlike what other studies and teaching experience have reported.
\end{abstract}

Key words: education, self-efficacy, teachers, gender, experience.

\section{RESUMO}

Consideração às complexas demandas emanadas da escola, da política e da sociedade, o artigo tem o propósito de descrever os sentimentos de auto-eficácia nos desempenhos profissionais, em uma amostra de 544 professores

La investigación que se presenta en este artículo emana de la tesis doctoral Sentimiento de Autoeficacia en una Muestra de Profesores Chilenos. 
chilenos. Os estudos do constructo, realizados por quase três décadas, não só tentaram explicar a realidade educativa, mas também valorizado o importante papel que assumem nas práticas pedagógicas. Neste sentido, foi realizada a tradução e a adaptação do questionário "Teachers' Sense of Efficacy Scale" (TSES) de TschannenMoran e Woolfolk-Hoy (2001). Ressalta-se que o processo de validação revelou índices de interesse tanto para estrutura fatorial como para sua confiabilidade. Resultados demonstraram que o gênero não é uma variável que altera os sentimentos de auto-eficácia docente, ao contrário da experiência docente, que apresentou diferenças significativas

Palavras chave: educação, auto-eficácia, professores, gênero, experiência.

\section{INTRODUCCIÓN}

La teoría de autoeficacia ha sido motivo de estudio en diversos ámbitos del funcionamiento humano como la política, la economía, el deporte, la salud y la educación. Atendiendo al último ámbito indicado, ha surgido la necesidad de identificar y comprender los sentimientos de autoeficacia que poseen los profesores en el contexto educativo chileno. El estudio de dichos sentimientos constituye un área de información valiosa acerca de cuán capaces se sienten los docentes para ejecutar las tareas de enseñanza y generar el aprendizaje en los estudiantes (Schunk \& Pajares, 2004).

La literatura ha dado cuenta del impacto de estos sentimientos en el favorecimiento de procesos educativos enriquecedores en profesores y en estudiantes. Del mismo modo, su influencia resulta poderosa en los desempeños pedagógicos, ya sea facilitando o entorpeciendo el aprendizaje y el logro en los estudiantes. En efecto, los estudios avalan que positivos sentimientos de autoeficacia fomentan el uso de variadas estrategias de enseñanza y aprendizaje, la adaptación de la enseñanza a las singularidades educativas de los aprendices, el manejo eficaz de los conflictos de aula y la promoción de la participación en los estudiantes.

Atendiendo a lo anterior, las escuelas deben enfocar sus prácticas de enseñanza hacia el fomento del desarrollo intelectual, y de la motivación intrínseca en los estudiantes, con tal que adquieran las competencias para aprender durante toda la vida. Por esto es imprescindible que los profesores cuenten con férreos sentimientos de autoeficacia, para que los ambientes escolares sean positivos, productivos y favorecedores del desarrollo cognitivo y emocional en los aprendices.

\section{LAS BASES TEÓRICAS DE LA AUTOEFICACIA}

La teoría de autoeficacia ha pasado por distintas etapas conceptuales y metodológicas. Desde sus comienzos hasta la actualidad, esta teoría ha intentado demostrar cómo los aspectos cognitivos, conductuales, contextuales y afectivos de las personas están condicionados por la autoeficacia. Albert Bandura (1977), su máximo impulsor, dio forma y consistencia a su teoría, al considerar que el pensamiento autorreferente actúa mediando la conducta y la motivación en las personas.

Asimismo, destacó - de manera sostenida- la presencia de dos tipos de expectativas: una de eficacia definida como el convencimiento de que una ejecución se realice con éxito, y otra de resultados que no es más que la estimación de los resultados a conseguir una vez realizada la ejecución. Del mismo modo, enfatizó que el sentimiento de autoeficacia puede 
ser desarrollado a través de cuatro fuentes: las experiencias de dominio, las experiencias vicarias, la persuasión social y los estados psicológicos y emocionales (Bandura, 1999).

El impacto de estos factores marca profundas diferencias en las personas con un fuerte o un débil sentimiento de autoeficacia. Las primeras poseen un interés intrínseco que las hace esforzarse y perseverar pese a las dificultades, son capaces de visualizar los problemas como desafíos y oportunidades de aprendizaje y, por ende, de progreso en sus habilidades. Las segundas, en cambio, carecen de compromiso, flaquean rápidamente ante las adversidades, evitan tareas complejas, huyen de los problemas y dejan de creer en sí mismas.

\subsection{DOS PERSPECTIVAS PARA EL ESTUDIO DE LA AUTOEFICACIA: ROTTER Y BANDURA}

Para comprender la teoría de autoeficacia en el profesor, resulta imprescindible exponer las dos perspectivas que han impulsado su estudio. La primera deriva desde Julian Rotter y la otra deriva desde Albert Bandura.

Bajo la perspectiva de la teoría del aprendizaje social de Rotter (1966), surgió la primera línea de investigación sobre la eficacia del profesor. Los estudios provenientes de esta perspectiva consideraron que la eficacia del profesor, junto con albergar una expectativa generalizada en torno a las consecuencias internas o externas de las conductas, constituía un rasgo relativamente estable en torno a las capacidades para ejercer control sobre sus conductas pedagógicas.

La internalidad o externalidad, como expectativa generalizada, actúa principalmente pronosticando e informando acerca de cómo las personas conciben y estructuran sus experiencias. A partir de esto, Rotter (1966) estableció dos tipologías: [LOC] interno y externo. Así, cuando las personas tienen un lugar de control interno, asumen las responsabilidades de sus circunstancias, involucrándose en situaciones donde el éxito lo alcanzan por sus capacidades y esfuerzos. En contraste, las personas con un lugar de control externo aceptan que su vida está controlada por personas o fuerzas externas y, por lo tanto, los logros o fracasos que puedan obtener los atribuyen al azar o a la suerte (Woolfolk, 2006).

Un segundo enfoque para el estudio de autoeficacia del profesor provino de la teoría socio-cognitiva de Bandura (1977). Este psicólogo afirma que la autoeficacia está relacionada con las capacidades personales para identificar las oportunidades que ofrece el entorno e interpretar los obstáculos que se perciben. De ahí que la autoeficacia no constituya un rasgo estable de la personalidad, por el contrario, tiene que ver con un juicio de capacidad donde "no se es capaz para todo, ni a los mismos niveles" (Garrido, 2000: 13). Por otra parte, la capacidad de predicción de comportamientos futuros tiene mayor éxito con la autoeficacia que con el lugar de control. Como evidencia de esto, es importante mencionar el meta-análisis de Stajkovic \& Luthans (1998), quienes predijeron que un $72 \%$ de las personas con alta autoeficacia tendrían un mejor rendimiento, frente a los que obtienen una menor puntuación.

Producto de lo antes dicho, y durante las etapas iniciales del estudio de la autoeficacia docente, la incipiente conceptualización no tenía límites claros entre lo que aportaba Rotter o Bandura, lo cual acarreó serias dificultades para comprender y medir el constructo (Prieto, 2007). Las dificultades permanecieron hasta la década de los '90, con la diferencia de que la tendencia investigativa se amparó en los postulados de Bandura y en la preocupación por 
la construcción de medidas válidas y fiables que no han estado, sin embargo, exentas de críticas (Charalambous, Philippou \& Kyriakides, 2008; Ciani, Summers \& Easter, 2008). Actualmente, ni la producción ni las críticas han cesado, ni menos la consideración de que la eficacia del profesor constituye una clave influyente en el comportamiento profesional del docente y en el aprendizaje de los estudiantes (Klassen, Tze, Betts \& Gordon, 2011).

\subsection{CONCEPTUALIZACIÓN DEL SENTIMIENTO DE AUTOEFICACIA EN LOS PROFESORES}

Las complejas demandas emanadas de la escuela, la política y la sociedad impactan fuertemente en los sentimientos de autoeficacia y en las capacidades de los profesores para suscitar el aprendizaje en los estudiantes, y mantener el compromiso con su profesión (Klassen et al., 2009; Chan, 2008b). Conforme a ello, la cantidad de estudios que han intentado explicar la realidad educativa a través de la autoeficacia abarca casi tres décadas, valorando el importante papel que tiene en la conducta humana y, por ende, en la práctica pedagógica (Pajares, 2003; Bandura, 1987).

A juicio de varios expertos, la eficacia personal ejerce una poderosa influencia en los niveles de realización o logro de las personas (Pajares, 2006), mientras que TschannenMoran \& Johnson (2011) sostienen que la autoeficacia comporta una evaluación de las capacidades reales para lograr un rendimiento adecuado, en un ámbito de funcionamiento específico. De este modo, se podría indicar que la eficacia del profesor constituye un tipo de autoeficacia que contempla una evaluación de las propias capacidades para alcanzar un nivel adecuado de rendimiento en una tarea y un entorno específico. Sin embargo, para alcanzar un desempeño óptimo, los docentes deben manejarse en dos niveles de pensamiento: por un lado, sentirse capaces y, por otro, juzgarse capaces. La combinación de estos dos niveles indica conocimiento, despliegue y gestión de las competencias, habilidades o capacidades que poseen, de manera que el sentimiento de autoeficacia actúa como un mecanismo cognitivo mediador entre sus conocimientos y sus actos pedagógicos (Prieto, 2007; Bandura, 1987).

Aún más, Tschannen-Moran \& Woolfolk (2001) enfatizan en los sentimientos de autoeficacia como juicios de capacidad docente para enseñar y suscitar aprendizaje en determinadas y variadas circunstancias. Recientes aportes surgen de la opinión de Ross \& Bruce (2007), quienes conciben el sentimiento de autoeficacia del profesor como una expectativa que promueve el aprendizaje en los estudiantes. Otros consideran que la autoeficacia del profesor es la capacidad para realizar con éxito tareas específicas de enseñanza (Dellinger, Bobbett, Olivier \& Ellet, 2008). Por su parte, Pas, Bradshaw \& Hershfeldt (2012) opinan que este sentimiento está referido a la capacidad de los profesores de crear un ambiente adecuado para el aprendizaje.

A efectos de aclarar lo que se entiende por autoeficacia en el profesor y diferenciarla de la efectividad docente, Anita Woolfolk (Cit. en Shaughnessy, 2004) señala que las denominaciones más adecuadas podrían ser: sentido de eficacia docente, autoeficacia docente, eficacia en la enseñanza, creencias de eficacia docente y eficacia docente percibida. Igualmente, Torre (2007) manifiesta que las diferentes conceptualizaciones para un mismo constructo (tales como juicios, creencias, opiniones y sentimientos) no merecen objeción, aun cuando Bandura en sus escritos utiliza mayormente "creencias de autoeficacia" y/o “juicios de capacidad personal”. Por su parte, Bueno, Bermúdez y Martín (2009) indican que hablar de la autoeficacia es hablar de un sentimiento que actúa como mediador del conocimiento y la acción. 
Pese a que la denominación de "sentimiento de autoeficacia" no es ampliamente utilizada, podría ser la más adecuada para diferenciarla de lo que son las creencias y/o las teorías implícitas de los profesores. El sentimiento de autoeficacia abarca los planos cognitivos, afectivos y conductuales, mientras que las creencias y/o teorías implícitas se centran más en lo cognitivo, en relación con la naturaleza del conocimiento y del aprendizaje. Esta sutil diferencia podría explicar la relación positiva que existe entre ambos constructos, de acuerdo a resultados de las escasas investigaciones llevadas a cabo al respecto (Chrysostomou \& Philippou, 2010; Esterly, 2003).

Por otra parte, es necesario destacar que en una educación de calidad no basta con que los profesores tengan amplios conocimientos y habilidades necesarias para el cargo, sino que, además, deben ser poseedores de una férrea confianza y/o convicción en lo que hacen. Para Rizvi \& Elliot (2005), el sentimiento de autoeficacia del profesorado es parte sustancial de su profesionalidad, así como también lo son la práctica, el liderazgo y la colaboración. Un funcionamiento pedagógico eficaz para alcanzar un alto grado de destreza requiere el desarrollo de dos procesos: el crecer y el aprender (Artino, 2006). En la medida en que estén articulados cada uno de los elementos mencionados, los profesores podrán diseñar y organizar ambientes eficaces de enseñanza y aprendizaje (Philippou \& Christou, 2002).

Por último, conviene señalar que el sentimiento de autoeficacia en el profesorado está relacionado con otras variables también importantes. Entre ellas se encuentran el logro y la motivación de los estudiantes (Caprara, Barbaranelli, Steca \& Malone, 2006), el compromiso con el trabajo pedagógico y la satisfacción personal (Caprara, Barbaranelli, Borgogni \& Steca, 2003), el optimismo académico (Woolfolk, Hoy \& Kurz, 2008), las preocupaciones de la enseñanza (Boz \& Boz, 2010), las innovaciones educativas (Tschannen-Moran \& McMaster, 2009) y la sensibilidad cultural (Siwatu, 2007).

\subsection{LOS SENTIMIENTOS DE AUTOEFICACIA SEGÚN EL GÉNERO Y LA EXPERIENCIA}

La variable género ha sido una dimensión bastante compleja de captar, puesto que las investigaciones evidencian discrepancias. Así pues, en algunas muestras las profesoras tienen mayor eficacia que los profesores, mientras que en otras no arrojan diferencias significativas. Algunas razones de estas divergencias podrían explicarse por: (a) la calidad de las muestras abundantes de profesoras en los niveles primarios, y con una disminución en los niveles secundarios; (b) los roles de género culturalmente definidos que asumen la carrera docente como una profesión eminentemente femenina, y; (c) la tendencia a disminuir la diferencia en el sentimiento de autoeficacia en tanto aumenta el nivel escolar (Vera, Salanova \& Martín del Río, 2011; Fives \& Looney, 2009; Cheung, 2008, 2006; Gencer \& Cakiroglu, 2007).

Una evidencia clara de esta discrepancia surge de una investigación transcultural de las percepciones de eficacia entre profesores hindúes e iraníes, donde las mujeres tienen un nivel de eficacia mayor que los hombres. Incluso, como dato curioso, los profesores iraníes revelaron un sentimiento de autoeficacia mayor que sus pares hindúes (Sridhar \& Baidei, 2008). Por el contrario, Tschannen-Moran \& Johnson (2011) no encontraron diferencias significativas en la autoeficacia según el género, quizás por haber tomado una muestra principalmente femenina y predominantemente de Educación Primaria.

Otro aspecto importante a considerar en la eficacia del profesorado, de acuerdo al género, es la diferencia que puede evidenciar dicho sentimiento cuando se relaciona con la 
enseñanza de una disciplina, sea científica o humanista. A modo de ejemplo, Riggs (1991) indica que en la enseñanza de las ciencias, los profesores se sienten más autoeficaces que las profesoras, lo que estaría en concordancia con que la enseñanza de esta área se relaciona más con una participación masculina que femenina (Olaz, 2003; Zeldin \& Pajares, 2000).

Por otro lado, la experiencia tiene un gran poder de influencia en los sentimientos de eficacia en los profesores. Según Bandura (1999), las experiencias de dominio, la influencia de modelos competentes y la formación continua, conforman un aliciente de los juicios de eficacia docente. Sumado a lo anterior, este sentimiento se establece tempranamente en los procesos de aprendizaje y/o formación y, una vez instalado, es estable y resistente al cambio. Por esta razón, los juicios de eficacia en los futuros profesores resultan mucho más influenciables y moldeables que en los más experimentados. En base a lo anterior, diversas investigaciones arrojan resultados contradictorios que imposibilitan llegar a un acuerdo respecto a cómo y cuándo la experiencia engorda o adelgaza el sentimiento de autoeficacia en el profesorado (Chrysostomou \& Philippou, 2010; Woolfolk \& Spero, 2005; Mulholland \& Wallace, 2001).

Por ejemplo, Wolters \& Daugherty (2007) evidencian que los profesores con más años de servicio tienen una mejor opinión de eficacia en cuanto a las prácticas de enseñanza y evaluación, así como también para mantener el orden y crear un clima adecuado para el aprendizaje. Un resultado no muy alejado es el de Mertler (2004), quien -al comparar futuros profesores con profesores en servicio- obtuvo que estos últimos presentaban una mejor opinión de eficacia que los nóveles en la evaluación de la alfabetización. Otro caso más revela, por ejemplo, que profesores chinos con mayor experiencia poseen un mayor sentimiento de autoeficacia (Chan, 2008a).

Al contrario, Tschannen-Moran \& Johnson (2011) indican que la experiencia docente no constituye una variable influyente en la eficacia para la enseñanza de la lectoescritura. En tanto, Gorrell \& Dharmadasa (1994) exponen que, mientras los futuros profesores reportan un mayor juicio de eficacia para implementar una nueva metodología de enseñanza, los profesores en servicio reportan este mismo juicio para la gestión del aula, la organización de la enseñanza y, por lo mismo, un mayor impacto en el aprendizaje de los estudiantes.

Por el contrario, otras investigaciones arrojan relaciones negativas entre experiencia y autoeficacia en el profesorado no sólo a nivel correlacional, sino también en relaciones lineales entre años de servicio en profesores de Primaria y Secundaria, por cuanto entre 0 a 23 años de experiencia se concentran los mayores niveles de autoeficacia, para luego disminuir paulatinamente (Klassen \& Chiu, 2010; Taylor \& Tashakkori, 1995; Hoy \& Woolfolk, 1993).

\section{METODOLOGÍA}

\subsection{OBJETIVO GENERAL}

Identificar el sentimiento de autoeficacia en una muestra de profesores en Chile.

\subsection{OBJETIVOS ESPECÍFICOS}

- Describir los sentimientos de autoeficacia en profesores chilenos en función del género y la experiencia docente. 
- Comparar los sentimientos de autoeficacia en profesores chilenos en función del género.

- Comparar los sentimientos de autoeficacia en profesores chilenos en función de la experiencia docente.

\subsection{HIPÓTESIS}

- Los sentimientos de autoeficacia de los profesores chilenos presentan diferencias según el género de los profesores.

- Los sentimientos de autoeficacia de los profesores chilenos presentan diferencias según su experiencia profesional.

\subsection{TIPO DE ESTUDIO}

La investigación realizada ha empleado una metodología cuantitativa no experimental y, por lo tanto, no han sido manipuladas las variables consideradas en el estudio. La tipología descriptiva y transeccional ha contemplado un estudio de encuesta donde los datos fueron recabados en un sólo momento con el propósito de analizarlos y describirlos (Hernández, Fernández y Baptista, 2010).

\subsection{MUESTRA}

La muestra está compuesta por 544 profesores (véase Tabla $\mathrm{N}^{\circ} 1$ ) de las áreas urbanas de las Provincias de Marga-Marga, Quillota y Valparaíso, que tienen desempeño en centros municipalizados (220), particulares subvencionados (262) y particulares pagados (62). Cabe señalar que -de esta muestra- 382 son mujeres y 162 son hombres, los que imparten clases en los niveles de enseñanza infantil (50), primaria (284) y secundaria (210). Además, poseen una experiencia docente fluctuante entre más de 21 años (231) y entre 0 a 5 años (111). La participación de los sujetos ha sido voluntaria, encontrándose todos ellos en situación laboral activa y en posesión de la correspondiente titulación.

Tabla $N^{\circ} 1$. Características de la muestra

\begin{tabular}{|c|c|c|c|c|c|}
\hline \multicolumn{6}{|c|}{ Variables } \\
\hline \multirow{2}{*}{ Género } & Femenino & Masculino & & & \\
\hline & $70,2 \%$ & $29,8 \%$ & & & \\
\hline \multirow{2}{*}{ Nivel Enseñanza } & Infantil & Primaria & Secundaria & & \\
\hline & $9,2 \%$ & $52,2 \%$ & $38,6 \%$ & & \\
\hline \multirow{2}{*}{$\begin{array}{l}\text { Titularidad } \\
\text { Centro }\end{array}$} & Público & Concertado & Privado & & \\
\hline & $40,4 \%$ & $48,2 \%$ & $11,4 \%$ & & \\
\hline \multirow{2}{*}{$\begin{array}{l}\text { Experiencia } \\
\text { (años) }\end{array}$} & $0-5$ & $6-10$ & 11-15 & $16-20$ & +20 \\
\hline & $20,4 \%$ & $15,4 \%$ & $12,9 \%$ & $8,8 \%$ & $42,5 \%$ \\
\hline
\end{tabular}

Fuente: elaboración propia. 


\subsection{RECOGIDA DE INFORMACIÓN}

Como procedimiento para la obtención de los datos se utilizó la "encuesta postal" (Torrado, 2009), utilizando el correo institucional para hacer llegar a cada profesor las encuestas que debían cumplimentar. Los motivos de elección de este procedimiento son los siguientes: a) su menor costo permite la incorporación de más sujetos; b) los sesgos en las respuestas disminuyen, porque no está presente el investigador; c) las opiniones son entregadas en completo anonimato; d) favorece la llegada a poblaciones con difícil acceso, y; e) existe una mayor tranquilidad para que los sujetos respondan.

Para acceder a los centros educativos se contactó con sus administradores, con el fin de solicitar la autorización para llevar a cabo la investigación. Una vez conseguidas las correspondientes autorizaciones, se procedió a enviar a los profesores una carta de presentación y el cuestionario a cumplimentar.

\subsection{INSTRUMENTO}

El instrumento de medida seleccionado que mejor se adaptaba a las necesidades de esta investigación fue el "Teachers Self Efficacy Scale" (TSES) de Tschannen-Moran y Woolfolk (2001), en su versión larga. El instrumento consta de 24 ítems, puntuables en una escala de 1 a 9 (1 = "nada", 9 = "mucho"), que se dividen en 3 sub-escalas: a) "Eficacia para la implicación de los estudiantes", b) "Eficacia en las estrategias de enseñanza", y c) "Eficacia en el manejo de la clase". Posteriormente, se llevó a cabo el proceso de traducción y adaptación del TSES a la lengua española. El procedimiento utilizado para la traducción del inglés al español fue la traducción inversa o back translation (Martínez, Moreno y Muñiz, 2005).

Para validar el instrumento, 262 profesores de diferentes centros educativos, públicos, concertados y privados de las municipalidades de Valparaíso y Viña del Mar respondieron el instrumento con una escala tipo Likert simplificada de 1 ("nada") a 5 ("mucho"). El cambio de escala responde a las necesidades del contexto esgrimidas por los expertos y por los participantes de una prueba piloto previa de comprensión, que consideraron que una escala con más opciones podría ocasionar confusiones.

Las técnicas estadísticas seleccionadas que mejor se ajustaban a los datos para llevar a cabo la validación del constructo han sido el análisis factorial exploratorio y el índice de consistencia interna alfa de Cronbach. La primera técnica estadística reveló la presencia de un cuarto factor denominado "Eficacia en la atención a la singularidad de los estudiantes" (factor D), mientras que la segunda técnica arrojó, para el instrumento en general, un índice de consistencia interna de .92 . Pese a que los resultados no coinciden con la solución original del instrumento ( 3 factores con 24 ítems; 4 factores con 17 ítems) es posible destacar el 65,09\% de varianza total explicada y el logro de un alfa bastante alto, lo que hace que el instrumento sea válido y fiable (Covarrubias, 2014).

\section{RESULTADOS}

A fin de dar respuesta al objetivo sobre la descripción de los sentimientos de autoeficacia en los profesores chilenos, se consideraron las medidas descriptivas de las puntuaciones globales, así como los resultados de los cuatro factores del instrumento. Cabe señalar que 
dichos resultados, expuestos en las Tablas $\mathrm{N}^{\circ} 2$ y $\mathrm{N}^{\circ} 3$, son bastantes similares, salvo excepciones en las variables estudiadas.

Tabla $N^{\circ}$ 2. Estadísticos Descriptivos Media $(\mathrm{X})^{1}$

\begin{tabular}{|c|c|c|c|c|c|c|c|}
\hline \multirow{2}{*}{ 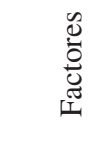 } & \multicolumn{2}{|c|}{ Género } & \multicolumn{5}{|c|}{ Experiencia Docente } \\
\hline & M & $\mathrm{H}$ & $0-5$ & 6-10 & $11-15$ & $16-20$ & +21 \\
\hline FA & 4,30 & 4,35 & 4,32 & 4,41 & 4,20 & 4,25 & 4,32 \\
\hline FB & 4,47 & 4,50 & 4,50 & 4,61 & 4,37 & 4,44 & 4,47 \\
\hline FC & 4,26 & 4,30 & 4,22 & 4,38 & 4,14 & 4,23 & 4,31 \\
\hline FDC" & 4,29 & 4,29 & 4,28 & 4,38 & 4,16 & 4,25 & 4,31 \\
\hline Global & 4,33 & 4,36 & 4,32 & 4,44 & 4,21 & 4,29 & 4,35 \\
\hline
\end{tabular}

Fuente: elaboración propia.

Tabla $N^{\circ}$ 3. Estadísticos Descriptivos Desviación Típica (D.T)²

\begin{tabular}{|c|c|c|c|c|c|c|c|}
\hline \multirow{2}{*}{ 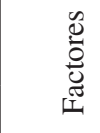 } & \multicolumn{2}{|c|}{ Género } & \multicolumn{5}{|c|}{ Experiencia Docente } \\
\hline & M & $\mathrm{H}$ & $0-5$ & $6-10$ & $11-15$ & $16-20$ & +21 \\
\hline FA & 0,52 & 0,52 & 0,50 & 0,51 & 0,56 & 0,50 & 0,51 \\
\hline FB & 0,45 & 0,47 & 0,44 & 0,44 & 0,44 & 0,57 & 0,44 \\
\hline $\mathrm{FC}$ & 0,56 & 0,58 & 0,58 & 0,56 & 0,51 & 0,69 & 0,54 \\
\hline FDC” & 0,56 & 0,53 & 0,56 & 0,55 & 0,59 & 0,73 & 0,50 \\
\hline Global & 0,44 & 0,44 & 0,42 & 0,44 & 0,42 & 0,56 & 0,42 \\
\hline
\end{tabular}

Fuente: elaboración propia.

Considerando los resultados de los estadísticos descriptivos según la variable Género, las Tablas $\mathrm{N}^{\circ} 2$ y $\mathrm{N}^{\circ} 3$ evidencian que la distribución del puntaje global y de los distintos

$* \mathrm{M}=$ Mujer; $\mathrm{H}=$ Hombre.

**FA = Eficacia en la implicación de los estudiantes; FB = Eficacia en las estrategias de instrucción; FC = Eficacia en el manejo de clase; FD = Eficacia en la atención a la singularidad de los estudiantes.

$2 * \mathrm{M}=$ Mujer; $\mathrm{H}=$ Hombre.

**FA = Eficacia en la implicación de los estudiantes; FB = Eficacia en las estrategias de instrucción; FC = Eficacia en el manejo de clase; FD = Eficacia en la atención a la singularidad de los estudiantes. 
factores del instrumento, cumplimentado por los 544 profesores chilenos, resultan ser bastantes similares no sólo en hombres, sino que también en mujeres. Esto quiere decir que tanto los profesores como las profesoras se sienten altamente eficaces para implicar a los estudiantes, para utilizar diversas estrategias de enseñanza y aprendizaje, para manejar la disciplina del aula, y para atender las necesidades educativas de los estudiantes.

Por su parte, los resultados descriptivos para la variable Experiencia Docente (véanse las Tablas $\mathrm{N}^{\circ} 2 \mathrm{y} \mathrm{N}^{\circ} 3$ ) revelan que para todos los rangos de Experiencia Docente el puntaje global y los puntajes para los distintos factores considerados en el instrumento presentan un valor promedio mayor en los profesores con una experiencia entre 6 a 10 años. En contraste con lo anterior, las puntuaciones de los profesores con una trayectoria pedagógica de 11 a 15 años reflejan valores promedios menores.

Paralelamente, los objetivos enfocados a comparar los sentimientos de autoeficacia de los profesores según el Género y la Experiencia docente fueron abordados con técnicas estadísticas paramétricas para la comparación de medias. Dichas comparaciones se hicieron tanto en términos globales como específicos de los factores que componen el cuestionario aplicado.

Tabla $N^{\circ}$ 4. Análisis de Varianza (ANOVA) $)^{3}$

\begin{tabular}{|c|c|c|c|c|}
\hline \multirow{2}{*}{ 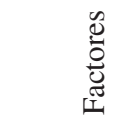 } & \multicolumn{2}{|c|}{ Género } & \multicolumn{2}{|c|}{ Experiencia Docente (años) } \\
\hline & $\mathrm{F}$ & Valor-p & $\mathrm{F}$ & Valor-p \\
\hline FA & 1,034 & 0,310 & 1,727 & 0,142 \\
\hline FB & 0,412 & 0,521 & 2,963 & $0,019^{*}$ \\
\hline $\mathrm{FC}$ & 0,591 & 0,442 & 2,374 & 0,051 \\
\hline FDC" & 0,001 & 0,977 & 1,621 & 0,168 \\
\hline Global & 0,537 & 0,464 & 2,770 & $0,027 *$ \\
\hline
\end{tabular}

Fuente: elaboración propia.

Como puede visualizarse en la Tabla $\mathrm{N}^{\circ} 4$, el resultado del valor-p asociado al estadístico de prueba $\mathrm{F}$ es mayor que 0,05 en la variable Género, por lo cual, es posible indicar que no hay diferencias estadísticamente significativas en las medias de los puntajes de los sentimientos de autoeficacia de profesores chilenos encuestados. Por lo tanto, la hipótesis de investigación no se ve confirmada.

Sin embargo, las medias de los puntajes en la variable Experiencia Docente no sólo advierten diferencias estadísticamente significativas, corroboradas por la aplicación del test de comparaciones múltiples de Tuckey Tipo b (véase Tabla $\mathrm{N}^{\circ}$ 5), sino que, además, confirman la segunda hipótesis de investigación.

$* \mathrm{p}<0,05$ 
Tabla $N^{\circ}$ 5. Subconjuntos Homogéneos según Test de Tuckey b

\begin{tabular}{|c|c|c|c|c|c|}
\hline \multicolumn{3}{|c|}{ Puntaje Global } & \multicolumn{3}{c|}{ Factor B } \\
\hline $\begin{array}{c}\text { Experiencia } \\
\text { Docente (años) }\end{array}$ & Subconjunto 1 & Subconjunto 2 & $\begin{array}{c}\text { Experiencia } \\
\text { Docente (años) }\end{array}$ & Subconjunto 1 & Subconjunto 2 \\
\hline 11 a 15 & 4,21 & & 11 a 15 & 4,37 & \\
\hline 16 a 20 & 4,29 & 4,29 & 16 a 20 & 4,44 & 4,44 \\
\hline 0 a 5 & 4,32 & 4,32 & 0 a 5 & 4,50 & 4,50 \\
\hline Más de 21 & 4,35 & 4,35 & Más de 21 & 4,46 & 4,46 \\
\hline 6 a 10 & & 4,44 & 6 a 10 & & 4,61 \\
\hline
\end{tabular}

Fuente: elaboración propia.

En consideración de lo anterior, la variable Experiencia Docente reveló diferencias significativas en la puntuación global y en el Factor B (Eficacia en las Estrategias de Instrucción) del instrumento. En concreto, los docentes con una experiencia profesional de 6 a 10 años albergan mayores sentimientos de autoeficacia que el profesorado con 11 a 15 años de trayectoria, en los cuatro factores del instrumento. Asimismo, ambos tramos también presentaron diferencias estadísticamente significativas en el Factor B del instrumento, lo que manifiesta que los profesores del primer tramo poseen mayores sentimientos de autoeficacia en el manejo de las estrategias de instrucción.

\section{DISCUSIÓN}

Para poder describir los sentimientos de autoeficacia en los profesores chilenos, se han considerado las medias de las puntuaciones globales y específicas del cuestionario. Así, un puntaje de tres puntos ha sido catalogado como neutral, mientras que aquellos que puntúan sobre o bajo dicho puntaje se catalogaron como altos y/o bajos niveles de autoeficacia, respectivamente (Swackhamer, Koellner, Basile \& Kimbrough, 2009; Palmer, 2006). Por su parte, los Análisis de Varianza (ANOVA) ejecutados se han encargado de la comparación de medias entre los sentimientos de autoeficacia con cada una de las variables de estudio por separado (Género y Experiencia), los cuales han revelado diferencias estadísticamente significativas sólo para la última variable.

Al describir los sentimientos de autoeficacia según el género de los profesores, los resultados han sido bastante similares, lo cual permite manifestar que tanto los profesores como las profesoras albergan niveles semejantes en sus sentimientos de autoeficacia. Asimismo, la comparación de medias entre sentimientos de autoeficacia y el género de los profesores chilenos no ha arrojado diferencias estadísticamente significativas y, por lo mismo, no se cumple la hipótesis de investigación. Ante ello, ambos géneros se sienten capaces, a un mismo nivel, para "Implicar", "Enseñar", "Manejar" y "Atender" a los estudiantes diariamente en los contextos educativos.

Cabe señalar que los expertos han esgrimido que el género es una variable bastante compleja de captar, porque para algunas investigaciones sí ha evidenciado diferencias y para 
otras no (Fives \& Looney, 2009; Sridhar \& Baidei, 2008; Gencer \& Cakiroglu, 2007). En lo que respecta a este estudio, los resultados resultan coincidentes con aquellos que no reportan diferencias en el sentimiento de autoeficacia entre hombres y mujeres docentes (TschannenMoran \& Johnson, 2011). Independientemente de ello, y complementando lo antes dicho, los resultados obtenidos podrían estar sesgados por una estructura de muestra principalmente femenina, la cual alcanza el 70,2\% y, además, por los roles de género culturalmente adquiridos, los cuales hacen de la profesión docente una carrera femenina (Ávalos, 2003).

En cuanto a la experiencia y su impacto en los sentimientos de autoeficacia docente, curiosamente los profesores en el tramo de 6 a 10 años reportaron una alta autoeficacia para las estrategias de enseñanza y aprendizaje. Al mismo tiempo, los docentes con una trayectoria de 11 a 15 años evidenciaron niveles de autoeficacia menores para todos los factores del instrumento.

Por si fuera poco, los resultados de la comparación de medias para el puntaje global han arrojado diferencias estadísticamente significativas para el tramo de 6 a 10 años, con respecto al de 11 a 15 años. Incluso, al desglosar los factores, es posible apreciar que las diferencias se presentan en el Factor B ("Eficacia en las estrategias de enseñanza y aprendizaje"). Todo esto confirma la hipótesis de investigación en cuanto a la presencia de diferencias significativas entre los sentimientos de autoeficacia y la experiencia profesional del docente.

Resulta evidente que los resultados de esta investigación no coinciden con los de otras investigaciones $\mathrm{y}$, por lo mismo, es arbitrario poder aseverar si la experiencia actúa positiva o negativamente en los sentimientos de autoeficacia docente. Más bien, los resultados de este estudio, en una muestra de profesores chilenos, constituyen un antecedente al no acuerdo de la experiencia como catalizadora en el aumento o disminución de la autoeficacia. Para variados investigadores, estos sentimientos son menores en los profesores nóveles y mayores en los profesores más experimentados (Tschannen-Moran \& Woolfolk, 2007; Wolters \& Daugherty, 2007; Cheung, 2006), para otros no existen diferencias entre ellos, con la salvedad de que, a partir de los 10 años de servicio, la experiencia los haría sentir más eficaces (Guo, Justice, Sawyer \& Tompkins, 2011; Tschannen-Moran \& Johnson, 2011; Fives \& Buehl, 2010).

Independientemente de los debates sobre el impacto de la experiencia en los sentimientos de autoeficacia en los profesores, es posible indicar que los resultados para esta investigación no son ni generalizables ni tampoco concluyentes. Es probable que este peculiar comportamiento se haya producido por la captura heterogénea de la experiencia en los participantes, la cual ha provocado que las diferencias significativas sólo las representen el 15,4\% (6 a 10 años) y el 12,8\% (11 a 15 años) de la población docente encuestada. Aún más, las diferencias significativas entre ambos tramos podrían estar explicadas por la etapa profesional intermedia en la cual están situados, caracterizada en parte por el cuestionamiento de sus quehaceres pedagógicos y por la flexibilidad de sus sentimientos de autoeficacia.

\section{CONCLUSIONES}

La presente investigación ha permitido explorar el comportamiento de la teoría de autoeficacia $\mathrm{y}$, por ende, los sentimientos que emanan de ella en una muestra de profesores chilenos, colaborando con la proyección de futuros trabajos. Además, se alinea con los estudios nacionales enfocados tanto en los sentimientos de autoeficacia docente, así como de aquellos 
que están siendo formados para educar (Ávalos y Sotomayor, 2012; Tagle, Del Valle y Flores, 2012; Araya, Taut, Santelices y Manzi, 2011; Del Río, Lagos y Walker, 2011; Montecinos, Barrios y Tapia, 2011; Ávalos, Cavada, Pardo y Sotomayor, 2010; Guerra, 2008).

$\mathrm{Al}$ describir los sentimientos de autoeficacia desde la perspectiva del género de los profesores, los resultados han sido bastantes similares, lo cual permite manifestar que tanto los profesores como las profesoras albergan niveles semejantes en sus sentimientos de autoeficacia. Incluso, la comparación de medias entre sentimientos de autoeficacia y el género de los profesores chilenos no ha arrojado diferencias estadísticamente significativas y, por lo mismo, no se cumple la hipótesis de investigación. Ante ello, ambos géneros se sienten capaces, a un mismo nivel, para "Implicar", "Enseñar", "Manejar" y "Atender" diariamente a los estudiantes en los contextos educativos.

En cuanto a la experiencia y su impacto en los sentimientos de autoeficacia docente, curiosamente los profesores en el tramo de 6 a 10 años reportaron una alta autoeficacia para las estrategias de enseñanza y aprendizaje. Por otro lado, los docentes con una trayectoria de 11 a 15 años evidenciaron niveles de autoeficacia menores para todos los factores del instrumento. Por si fuera poco, los resultados de la comparación de medias para el puntaje global han arrojado diferencias estadísticamente significativas para el tramo de 6 a 10 años con respecto al de 11 a 15 años. Más aún, al desglosar los factores es posible apreciar que las diferencias se presentan concretamente en el Factor B ("Eficacia en las estrategias de enseñanza y aprendizaje"). Todo esto confirma la hipótesis de investigación en cuanto a la presencia de diferencias significativas entre los sentimientos de autoeficacia y la experiencia profesional del docente.

Finalmente, es necesario señalar que estudiar los sentimientos de autoeficacia en una muestra de profesores chilenos no sólo ha constituido un avance en el conocimiento de las opiniones que tienen acerca de sus capacidades, sino que también ha supuesto una aportación a la comprensión de que parte de los resultados educativos podría estar explicada por este constructo. En efecto, los mencionados sentimientos actúan como mediadores de la acción docente en el aula, impactando tanto en las trayectorias profesionales de los docentes, como en las trayectorias de aprendizaje de los estudiantes.

\section{REFERENCIAS BIBLIOGRÁFICAS}

Araya, C., Taut, S., Santelices, V. y Manzi, J. (2011). Validez consecuencial del programa de asignación de excelencia pedagógica en Chile. Estudios Pedagógicos, vol.37 (2), 25-42.

Artino, A. R. (2006). Self-efficacy Beliefs: From Educational Theory to Instructional Practice. Retrieved on April 19, 2011 from http://www.ucm.es/BUCM/checkip.php?/docview/62008727?acc ountid $=14514$

Ávalos, B. y Sotomayor, C. (2012). Cómo ven su identidad los docentes chilenos. Perspectiva Educacional, vol.51 (1), 77-95.

., Cavada, P., Pardo, M. y Sotomayor, C. (2010). La profesión docente: Temas y discusiones en la literatura internacional. Estudios Pedagógicos, vol.36 (1), 235-263.

(2003). La formación docente inicial en Chile. Recuperado el 17 de julio de 2010 desde http://www.oei.es/docentes/info_pais/informe_formacion_docente_chile_iesalc.pdf

Bandura, A. (1999). Ejercicio de la eficacia personal y colectiva en sociedades cambiantes. En A. Bandura (Ed.), Autoeficacia: Cómo afrontamos los cambios de la sociedad actual (pp.19-54). Bilbao: Descleé De Brouwer. 
Estudios Pedagógicos XLI, N 1: 63-78, 2015

SENTIMIENTO DE AUTOEFICACIA EN UNA MUESTRA DE PROFESORES CHILENOS DESDE LAS PERSPECTIVAS DE GÉNERO Y EXPERIENCIA

(1987). Pensamiento y acción. Fundamentos sociales. Barcelona: Martínez Roca.

(1977). Self-efficacy: Toward a Unifying Theory of Behavioral Change. Psychological

Review, vol.84 (2), 191-215.

Boz, Y. \& Boz, N. (2010). The Nature of the Relationship between Teaching Concerns and Sense of Efficacy. European Journal of Teacher Education, vol.33 (3), 279-291.

Bueno, J. A., Bermúdez, T. y Martín, E. (2009). El profesor y el sentimiento de autoeficacia en el ámbito de las competencias del autoconocimiento personal. International Journal of Developmental and Educational Psychology, vol.2 (2), 681-690.

Caprara, G. V., Barbaranelli, C., Steca, P. \& Malone, P. S. (2006). Teachers' Self-efficacy Beliefs as Determinants of Job Satisfaction and Students' Academic Achievement: A Study at the School Level. Journal of School Psychology, vol.44 (6), 473-490.

Borgogni, L. \& Steca, P. (2003). Efficacy Beliefs as Determinants of Teachers' Job Satisfaction. Journal of Educational Psychology, vol.95 (4), 821-832.

Chan, D. (2008a). Dimensions of Teacher Self-efficacy among Chinese Secondary School Teachers in Hong Kong. Educational Psychology, vol.28 (2), 181-194. (2008b). Emotional Intelligence, Self-efficacy, and Coping among Chinese Prospective and In-service Teachers in Hong Kong. Educational Psychology, vol.28 (4), 397-408.

Charalambous, C., Philippou, G. \& Kyriakides, L. (2008). Tracing the Development of Preservice Teachers' Efficacy Beliefs in Teaching Mathematics during Fieldwork. Educational Studies in Mathematics, vol.67 (2), 125-142.

Cheung, H. Y. (2008). Teacher Efficacy: A Comparative Study of Hong Kong and Shanghai Primary In-service Teachers. The Australian Educational Researcher, vol.35 (1), 103-123. (2006). The Measurement of Teacher Efficacy: Hong Kong Primary In-service Teachers.

Journal of Education for Teaching, vol.32 (4), 435-451.

Chrysostomou, M. \& Philippou, G. (2010). Teachers' Epistemological Beliefs and Efficacy Beliefs about Mathematics. Procedia Social and Behavioral Sciences, vol.9, 1509-1515.

Ciani, K. D., Summers, J. J. \& Easter, M. A. (2008). A “Top-down” Analysis of High School Teacher Motivation. Contemporary Educational Psychology, vol.33 (4), 533-560.

Covarrubias, C. G. (2014). El sentimiento de autoeficacia en una muestra de profesores chilenos. Tesis Doctoral. Universidad Complutense de Madrid, España.

Del Río, F., Lagos, C. y Walker, H. (2011). El efecto de las experiencias de práctica en el desarrollo del sentido de autoeficacia en la formación inicial de educadoras de párvulos. Estudios Pedagógicos, vol.37 (1), 149-166.

Dellinger, A. B., Bobbett, J. J., Olivier, D. F. \& Ellet, C. D. (2008). Measuring Teachers' Selfefficacy Beliefs: Development and Use of the TEBS-Self. Teaching and Teacher Education, vol.24 (2), 751-766.

Esterly, E. (2003). A Multi-method Exploration of the Mathematics Teaching Efficacy and Epistemological Beliefs of Elementary Pre-service and Novice Teachers. PhD Thesis. Ohio State University, United States.

Fives, H. \& Buehl, M. M. (2010). Examining the Factor Structure of the Teachers' Sense of Efficacy Scale. The Journal of Experimental Education, vol.78 (1), 118-134.

\& Looney, L. (2009). College Instructors' Sense of Teaching and Collective Efficacy. International Journal of Teaching and Learning in Higher Education, vol.20 (2), 182-191.

Garrido, E. (2000). Autoeficacia en el mundo laboral. Apuntes de Psicología, vol.18 (1), 19-38.

Gencer, A. S. \& Cakiroglu, J. (2007). Turkish Pre-service Science Teachers' Efficacy Beliefs regarding Science Teaching and their Beliefs about Classroom Management. Teaching and Teacher Education, vol.23 (5), 664-675.

Gorrell, J. \& Dharmadasa, K. H. (1994). Perceived Self-efficacy of Pre-service and in Sri Lankan Teachers. International Education, vol.24 (1), 23-36.

Guerra, P. (2008). Creencias epistemológicas y de eficacia docente de profesores que postulan 
al programa de acreditación de excelencia pedagógica y su relación con las prácticas de aula. Tesis de Magíster. Pontificia Universidad Católica, Chile. Recuperada el 12 de septiembre de 2010 desde http://uc-cl.academia.edu/paulaguerra/Books/700783/Creencias_epistemologicas_y_ de_eficacia_docente_de_profesores_que_postulan_al_programa_de_acreditacion_de_excelencia_ pedagogica_y_su_relacion_con_las_practicas_de_aula._Tesis_de_Magister_en_Psicologia_ Educacional_Pontificia_Universidad_Catolica_de_Chilee

Guo, Y., Justice, L., Sawyer, B. \& Tompkins, V. (2011). Exploring Factors related to Preschool Teachers' Self-efficacy. Teaching and Teacher Education, vol.27 (5), 961-968.

Hernández, R., Fernández, C. y Baptista, P. (2010). Fundamentos de metodología de la investigación. $2^{\text {da }}$ Edición. Madrid: McGraw-Hill.

Hoy, W. \& Woolfolk, A. (1993). Teachers' Sense of Efficacy and the Organizational Health of School. Elementary School Journal, vol.93 (4), 355-372.

Klassen, R. M., Tze, V. M. C., Betts, S. M. \& Gordon, K. A. (2011). Teacher Efficacy Research 19982009: Signs of Progress or Unfulfilled Promise? Educational Psychological Review, vol.23 (1), 21-43. \& Chiu, M. M. (2010). Effects on Teachers' Self-efficacy and Job Satisfaction: Teacher Gender, Years of Experience, and Job Stress. Journal of Educational Psychology, vol.102 (3), 741-756. Bong, M., Usher, E. L., Chong, W., Huan, V. S., Wong, I. \& Georgiou, T. (2009). Exploring the Validity of a Teachers' Self-efficacy Scale in Five Countries. Contemporary Educational Psychology, vol.34 (1), 67-76.

Martínez, R., Moreno, R. y Muñiz, J. (2005). Construcción de los ítems. En J. Muñiz, A. Fidalgo, E. García Cueto, R. Martínez y R. Moreno (Eds.), Cuadernos de Estadística 30. Análisis de los ítems (pp. 9-52). Madrid: La Muralla.

Mertler, C. A. (2004). Secondary Teachers' Assessment Literacy: Does Classroom Experience Make a Difference? American Secondary Education, vol.33 (1), 49-64.

Montecinos, C., Barrios, C. y Tapia, M. (2011). Relación entre estilos de supervisión durante la práctica profesional y las creencias de autoeficacia de los estudiantes de Pedagogía en Educación Básica. Revista Perspectiva Educacional, vol.50 (2), 96-122.

Mulholland, J. \& Wallace, J. (2001). Teacher Induction and Elementary Science Teaching: Enhancing Self-efficacy. Teaching and Teacher Education, vol.17 (2), 243-261.

Olaz, F. (2003). Autoeficacia y diferencia de géneros. Aporte a la explicación del comportamiento vocacional. Revista de Psicología General, vol.56 (3), 359-376.

Pajares, F. (2006). Self-efficacy during Childhood and Adolescence. In F. Pajares \& T. Urdan (Eds.), Self-efficacy Beliefs of Adolescents. Vol. 5. (pp. 339-367). Greenwich, CT: Information Age. . (2003). Self-efficacy Beliefs, Motivation, and Achievement in Writing: A Review of the Literature. Reading and Writing Quarterly, vol.19 (2), 139-158.

Palmer, D. (2006). Durability of Changes in Self-efficacy of Pre-service Primary Teachers. International Journal of Science Education, vol.28 (6), 655-671.

Pas, E. T., Bradshaw, C. P. \& Hershfeldt, P. A. (2012). Teacher and School-level Predictors of Teacher Efficacy and Burnout: Identifying Potential Areas for Support. Journal of School Psychology, vol.50 (1), 129-145.

Philippou, G. N. \& Christou, C. (2002). A Study of Mathematics Teaching Efficacy Beliefs of Primary Teachers. In G. Leder, E. Pehkonen \& G. Toerner (Eds.), Beliefs: A Hidden Variable in Mathematics Education? (pp. 211-232). Dordrecht: Kluwer Academic Publishers.

Prieto, L. (2007). Autoeficacia del profesor universitario. Eficacia percibida y práctica docente. Madrid: Narcea.

Riggs, I. M. (1991). Gender Differences in Elementary Science Teacher Self-efficacy. Annual Meeting of the American Educational Research Association. Chicago, IL, United States.

Rizvi, M. \& Elliot, B. (2005). Teachers' Perceptions of their Professionalism in Government Primary Schools in Karachi, Pakistan. Asia Pacific Journal of Teacher Education, vol.33 (1), 35-52.

Ross, J. \& Bruce, C. (2007). Professional Development Effects on Teacher Efficacy: Results of 
Estudios Pedagógicos XLI, N 1: 63-78, 2015

SENTIMIENTO DE AUTOEFICACIA EN UNA MUESTRA DE PROFESORES CHILENOS DESDE LAS PERSPECTIVAS DE GÉNERO Y EXPERIENCIA

Randomized Field Trial. Journal of Educational Research, vol.101 (1), 50-60.

Rotter, J. B. (1966). Generalized Expectancies for Internal versus External Control of Reinforcement. Psychological Monographs, vol.80 (1), 1-28.

Schunk, D. H. \& Pajares, F. (2004). Self-efficacy in Education Revisited: Empirical and Applied Evidence. In D. McInerney \& S. Van Ettem (Eds.), Big Theories Revisited. Vol. 4. (pp. 115-138). Greenwich, CT: Information Age.

Shaughnessy, M. F. (2004). An Interview with Anita Woolfolk: The Educational Psychology of Teacher Efficacy. Educational Psychology Review, vol.16 (2), 153-176.

Siwatu, K. O. (2007). Pre-service Teachers' Culturally Responsive Teaching Self-efficacy and Outcome Expectancy Beliefs. Teaching and Teacher Education, vol.23 (7), 1086-1101.

Sridhar, Y. N. \& Baidei, H. R. (2008). Teacher Efficacy Beliefs: A Comparison of Teachers in India and Iran. Journal of Indian Academy of Applied Psychology, vol.34 (1), 81-89.

Stajkovic, A. D. \& Luthans, F. (1998). Self-efficacy and Work-related Performance: A Metaanalysis. Psychological Bulletin, vol.124 (2), 240-261.

Swackhamer, L., Koellner, K., Basile, C. \& Kimbrough, D. (2009). Increasing the Self-efficacy of In-service Teachers through Content Knowledge. Teacher Educations Quarterly, vol.36 (2), 63-78.

Tagle, T., Del Valle, R. y Flores, L. (2012). Las creencias de autoeficacia percibida de estudiantes de pregrado de pedagogía en inglés. Revista Iberoamericana de Educación, vol.58 (4), 1-12. Recuperado el 22 de abril de 2012 desde http://www.rieoei.org/rie_contenedor. php? numero $=4667 \&$ titulo $=$ Las $\% 20$ creencias $\% 2$ de $\% 20$ autoeficacia $\% 20$ percibida $\% 20 \mathrm{de} \% 20$ estudiantes\%20de\%20pregrado\%20de\%20pedagog\%EDa\%20en\%20ingl\%E9s

Taylor, D. L. \& Tashakkori, A. (1995). Decision Participation and School Climate as Predictors of Job Satisfaction and the Teachers' Sense of Efficacy. The Journal of Experimental Education, vol.63 (3), 217-230.

Torrado, M. (2009). Estudios de encuesta. En R. Bisquerra (Coord.), Metodología de la investigación educativa. $2^{\text {da }}$ Edición (pp. 231-257). Madrid: La Muralla.

Torre, J. C. (2007). Una triple alianza para un aprendizaje universitario de calidad. Madrid: Publicaciones de la Universidad Pontificia Comillas.

Tschannen-Moran, M. \& Johnson, D. (2011). Exploring Literacy Teachers' Self-efficacy Beliefs: Potential Sources at Play. Teaching and Teacher Education, vol.27 (4), 751-761.

\& McMaster, P. (2009). Sources of Self-efficacy: Four Professional Development Formats and their Relationship to Self-efficacy and Implementation of a New Teaching Strategy. The Elementary School Journal, vol.110 (2), 228-245.

$\&$ Woolfolk, A. (2007). The Differential Antecedents of Self-efficacy Beliefs of Novice and Experienced Teachers. Teaching and Teacher Education, vol.23 (6), 944-956.

(2001). Teacher Efficacy: Capturing an Elusive Construct. Teaching and Teacher Education, vol.17 (7), 783-805.

Vera, M., Salanova, M. \& Martín del Río, B. (2011). Self-efficacy among University Faculty: How to Develop an Adjusted Scale. Anales de Psicología, vol.27 (3), 800-807.

Wolters, C. A. \& Daugherty, S. G. (2007). Goal Structures and Teachers' Sense of Efficacy: Their Relation and Association to Teaching Experience and Academic Level. Journal of Educational Psychology, vol.99 (1), 181-193.

Woolfolk, A., Hoy, W. K. \& Kurz, N. (2008). Teacher's Academic Optimism: The Development and Test of a New Construct. Teaching and Teacher Education, vol.24 (4), 821-835. (2006). Psicología educativa. $9^{n a}$ Edición. México D.F.: Pearson-Educación. \& Spero, R. (2005). Changes in Teacher Efficacy during the Early Years of Teaching: A Comparison of Four Measures. Teaching and Teacher Education, vol.21 (4), 343-356.

Zeldin, A. L. \& Pajares, F. (2000). Against the Odds: Self-efficacy Beliefs of Women in Mathematical Scientific and Technological Careers. American Educational Research Journal, vol.37 (1), 215-246. 